\title{
Correlation Among Different Stages of Physiological Cycles and Sudden Sensorineural Hearing Loss in Female
}

\author{
Yuliang Zhao ${ }^{1}$, Cuiling Wang ${ }^{1}$, Jianping Wang ${ }^{1}$, and Xixing $\mathrm{Li}^{1}$ \\ ${ }^{1}$ Second Hospital of Hebei Medical University
}

June 8,2020

\begin{abstract}
Abstract: Objectives: Sudden deafness, also known as sudden sensorineural hearing loss (SSNHL), has numbers hypotheses about its etiology. One of them believes that changes in sex hormone levels may increase the risk of thrombosis and block the cochlear microcirculation, leading to the occurrence of SSNHL. Design: Women in different periods of physiological cycles, the levels of sex hormones in their body will change significantly, which had correlated to SSNHL. Participants: This study collected 435 female subjects who were admitted to the otolaryngology clinic of our hospital from July 2017 to November 2019. Setting: The participants were grouped by the menstrual cycle. Main outcome measures: The pure tone audiometry test results of the subjects were recorded and the differences among the groups were be analyzed. Results: 1 . There was a significant difference in the average hearing threshold of subjects in different periods $(\mathrm{F}(3,774)=8.773, \mathrm{P}<0.001)$, and the average hearing threshold in follicular period $(35.44 \mathrm{dBHL})$ was significantly lower than that in menstrual period $(51.09 \mathrm{dBHL}, \mathrm{P}<0.001)$, luteal period (42.69 dBHL, $\mathrm{P}=0.036)$ and menopause $(47.39 \mathrm{dBHL}, \mathrm{P}<0.001) ; 2$. There was a correlation between the subjects at different periods and the severity of SSNHL $(? 2=38.587, \mathrm{P}<0.001)$, and the SSNHL severity was the slightest in the follicular period according to the mean rank value. 3. The analysis of SSNHL typing of subjects at different periods were variously (?2=38.568, $\mathrm{P}<0.001)$. Conclusions: The results of this study can explain the effect of sex hormone on SSNHL to a certain extent, and we will further explore the relationship between sex hormone levels and SSNHL, in order to provide more support for the diagnosis and treatment of SSNHL.
\end{abstract}

\section{Background}

Sudden deafness refers to sudden and unexplained sensorineural hearing loss that occurs within 72 hours, and at least two adjacent frequencies have a hearing loss of [?] $20 \mathrm{dBHL}^{[1]}$, also known as sudden sensorineural hearing loss (SSNHL). The vast majority of SSNHL subjects are difficult to determine the apparent etiology, and the otolaryngologist generally thought to be the blood disorders in the inner ear, the dysfunction of the hair cells, or physiological structural damage of the inner ear. Up to now, various hypotheses had been proposed, mainly including: the hypothesis of inner ear blood supply disorder, the hypothesis of viral infection, the hypothesis of immunological factors and the hypothesis of sex hormone. Each etiology hypothesis can explain the pathogenesis of some subjects, but no etiology hypothesis can explain all cases.

The sex hormone hypothesis believes that changes in sex hormone levels may increase the risk of thrombosis and block the cochlear microcirculation, leading to the occurrence of SSNHL ${ }^{[2-4]}$. The menstrual cycle is a normal physiological cycle for women. In different stages of the menstrual cycle, the body's sex hormone levels vary significantly. Changes in sex hormone levels will cause various physiological changes, affecting the cardiovascular system, hematological system, endocrine system, etc., then changing the cochlear microcirculation and water-electrolyte balance, which in turn affect the entire auditory system. Women in different stages of the menstrual cycle are sure to have different occurrence and severity of SSNHL. However, 
there have not any clear report on the study of the differences in women's hearing at different menstrual stages. This study collected and analyzed the results of auditory examinations of female subjects in different menstrual cycles. By comparing the average hearing threshold value, the severity and typing of SSNHL, explored the correlation between the occurrence and severity of SSNHL and the menstrual cycle stage. The results of this study can explain the effect of sex hormone levels on SSNHL to a certain extent, and have a guiding role in the diagnosis and treatment of female SSNHL subjects.

\section{Materials and methods}

\subsection{General information}

In this study, 435 female subjects who were admitted to the otolaryngology clinic of our hospital between July 2017 and November 2019 were selected.

Inclusion criteria: female; diagnosed with SSNHL by the chief physician; age [?] 16 years and have menarche; age $<60$ years; can cooperate with pure tone audiometry.

Exclusion criteria: tympanic membrane perforation, otosclerosis and other nonsense neurological deafness; stroke, nasopharyngeal carcinoma, acoustic neuroma, autoimmune diseases, etc.; cannot collaborate with pure tone audiometry.

Due to the small number of "pregnancy" and "lactation" periods (5 and 7 respectively), and the hormones are more complex, this study do not consider them for the time being; 34 subjects had irregular menstruation narrated by themselves and could not determine their stage, which was also excluded. The remaining 389 subjects were divided into 32 people in the "menstrual period group, MP" (days 1-6 of the menstrual cycle), 57 people in the "follicular phase group, FP" (days 7-15 of the menstrual cycle), 144 people in the "luteal phase group, LP" (days 16-28 of the menstrual cycle) and 156 people in the "menopausal group, M".

\subsection{Checking Method}

Pure tone audiometry checking was used to have the pure tone thresholds. All the subjects sat in the standard audiometric room, the air and bone conducted pure tone auditory thresholds at frequencies of 250, 500, 1000, 2000, 4000 and $8000 \mathrm{~Hz}$ were examined by the professional technician. Then record and calculated the average hearing threshold. According to the "Otorhinolaryngology Head and Neck Surgery"

[5] and" Guidelines for Diagnosis and Treatment of Sudden Deafness" [1], determined the occurrence and severity of SSNHL (Normal: < 25 dBHL; Mild: 26-40 dBHL; Moderate: 41-55 dBHL; Moderately severe: 56-70 dBHL; Severe: 71-90 dBHL; Extremely severe:> 91 dBHL). And counted the number of the subjects of Low-frequency sudden deafness, High-frequency sudden deafness, Flat descending sudden deafness and Total sudden deafness respectively, according to the frequency and degree of hearing loss. Compared whether there were significant differences among the average hearing threshold, severity, and typing of subjects in different periods.

\subsection{Statistics}

Data analysis was performed using IBM SPSS Statistics 25 software. One-way analysis of variance was used for the average hearing threshold in different periods, and Tukey method was used for multiple comparisons. The severity of SSNHL in different periods was ordered data. Therefore the Kruskal-Wallis test in rank sum test was adopted. The typing was categorical data, using $\mathrm{R} \times \mathrm{C}$ table chi-square test, and further using Post hoc testing for in-depth analysis, according to adjusted standardized residuals to determine the differences among the groups: The 95\% CI boundary of standard normal distribution is 1.96 times the standard deviation, so when the absolute value of the adjusted standardized residual (ASR) is greater than 
1.96, the difference between the observed frequency and the expected frequency of this value is considered statistically significant. All diagrams were generated using Graph Prism 6.0 software.

\section{Results}

\subsection{One-way analysis of variance for the average hearing threshold of subjects in different periods}

The results showed that the average hearing threshold of menstrual, follicular, luteal and menopausal subjects were: $51.09 \mathrm{dBHL}, 35.44 \mathrm{dBHL}, 42.69 \mathrm{dBHL}, 47.39 \mathrm{dBHL} ; \mathrm{F}(3,774)=8.773, P<0.001$, indicating that there was a significant difference in the average hearing threshold of subjects in different periods. Further multiple comparisons test showed that the average hearing threshold of subjects in follicular phase was significantly lower than that in menstrual period $(P<0.001)$, luteal phase $(P=0.036)$ and menopause $(P<0.001)$. That is, subjects in follicular phase had better hearing than other periods (Figure 1 ,Table 1 ).

\subsection{The comparison of SSNHL severity of subjects in different periods}

Ranked tests of ordered data (Kruskal-Wallis test) were calculated among subjects with normal, mild, moderate, moderately severe, severe and extremely severe deafness in different periods. The results showed that $\chi^{2}$ $=38.587, \mathrm{df}=3, P<0.001$, indicating that the severity of SSNHL varies among subjects in different periods. The mean rank value showed that the SSNHL in the follicular phase has the least severe, that is, the hearing of subjects in the follicular phase was better than other periods (Table 2).

\subsection{Comparison of SSNHL typing of subjects in different periods}

According to the frequency and degree of hearing loss, the subjects in each periods were divided into four types: Low-frequency sudden deafness, High-frequency sudden deafness, Flat descending sudden deafness and Total sudden deafness, and $\mathrm{R} \times \mathrm{C}$ chi-square test was performed.

The results showed that $6.3 \%$ of the expected cell counts were lower than 5 , and the minimum expected count was 3.04, which met the requirements of chi-square test. Pearson $\chi^{2}=38.568$, df $=9, P<0.001$, indicating that the composition of SSNHL typing varies among subjects in different periods. There was a correlation between the periods and the SSNHL typing, and the correlation strength Cramer's $\mathrm{V}=0.182, P$ $<0.001$. Analysis of the significance of the difference according to the ASR of Post hoc testing.

The results show that: 1) subjects in menstrual period had more Flat descending sudden deafness and fewer High-frequency sudden deafness; 2) subjects in follicular period had more Low-frequency sudden deafness and fewer High-frequency sudden deafness; 3) subjects in luteal phase had more High-frequency sudden deafness; 4) menopause subjects had more Total sudden deafness; while, there was no significant differences in the comparison among other periods (Table 3).

\section{Discussion}

The incidence of SSNHL has been on the rise in recent years. Due to the lack of large-scale epidemiological investigations, the annual incidence can only be estimated to be about 5-20 / 100,000 people ${ }^{[6]}$. A multicenter study of SSNHL in China showed that there was no significant difference between the male and female ratios and the vast majority of cases were unilateral onset (the left side is slightly more than the right side) while very few bilateral incidences ${ }^{[7]}$. And some subjects had self-healing tendency ${ }^{[8]}$. The cause of this hearing loss was difficult to clear identification, and generally considered to include microvascular circulation disorders, allergic reactions, trauma, inflammation, tumors or genetic disorders. 
Some scholars had suggested that changes in hormone levels can affect the body's hearing in various ways. Hormonal fluctuation can change the composition of endolymph and perilymph, as well as ion transport process, causing mucosal and vascular hyperplasia, meanwhile suppressing immune response, leading to a potential viral infection ${ }^{[9]}$. Sex hormones had a regulatory effect on the central nervous system ${ }^{[10]}$, which can affect multiple systems such as breathing, circulation, and endocrine, causing complex physiological changes in the body, such as increasing the risk of thrombosis and blocking microcirculation in the cochlea, and further affecting hearing leads to the occurrence of SSNHL ${ }^{[11]}$; on the other hand, changes in sex hormone levels can cause lymphatic osmotic pressure disorder and sodium and potassium ion retention, which may affect the inner ear like Meniere ${ }^{[3]}$.

The menstrual cycle is the normal physiological cycle for female. In the initial stage of the menstrual cycle, the follicles in the ovary mature gradually regulated by the hypothalamus, and produce estrogen, which triggers ovulation. This stage is referred to as the "follicle / ovulation period". Then corpus luteum forms and secretes a large amount of progesterone, which suppresses the release of upstream gonadotropin-releasing hormone and gonadotropin through negative feedback, resulting in atrophy of the corpus luteum and a decrease in sex hormones. This stage is the "luteal phase". Without the support of sex hormones, the endometrium will undergo necrosis and shedding, resulting in menstrual cramps and entering the "menstrual period." Female in the menstrual period have faster body metabolism, higher oxygen consumption, increased cardiac output and blood volume, the mucosa will appear corresponding changes such as hyperemia and edema. Females ovaries and other gonads gradually decline with age, and the secretion of sex hormones decreases volatility. After perimenopause, sex hormones decrease sharply and enter "menopause period". Due to the special stages of menstrual cycle, pregnancy and menopause, the hormone levels of female individuals are more complicated and variable than those of male. Female in different stages of their menstrual cycles, the levels of sex hormones such as estrogen and progesterone fluctuate more drastically, so their hearing changes are also more complicated and problematic ${ }^{[12]}$.

Estrogen receptors are widely expressed in cochlear hair cells, spiral ganglia, vascular lines, spiral arteries, outer and inner hair cells, which suggested that the changes in auditory function and auditory symptoms in different physiological stages of women are related to the fluctuating changes of estrogen. It is generally believed that estrogen can excite neurons, improve nerve conduction, affect cochlear microvascular circulation and cochlear intra- and extra-lymphatic fluid ${ }^{[13]}$, and protect the hearing organs ${ }^{[14]}$. Progesterone receptors had not been observed in the cochlea, but progesterone can antagonize the excitatory effects of estrogen through other steroid receptors ${ }^{[15]}$. Due to the excitatory and protective effects of estrogen on the central nervous system ${ }^{[16,17]}$, auditory function would be more sensitive at the peak of estrogen, which shows that the hearing threshold of female with normal cycles in the follicular phase is significantly lower than that in the later luteal phase ${ }^{[18]}$. The pure tone audiometry results of our study also confirmed this aspect. Progesterone does the opposite, mainly to suppress and counteract the excitability of estrogen ${ }^{[19]}$. Our study showed that estrogen levels reach the peak during the follicular phase, and the average hearing threshold was significantly reduced at this time. Meanwhile, the severity of SSNHL in this period was also the slightest. The corpus luteum secretes large amounts of progesterone after formation, which results in a significant increase in the average hearing threshold of the subjects, causing the severity of SSNHL to be significantly worse than other periods. These all confirmed the effect of sex hormones on hearing.

Some studies had found that Otoacoustic emission (OAE) also fluctuates during the menstrual cycle, and the amplitude of Spontaneous otoacoustic emission (SOAE) during the follicular phase is greater than other stages. A study on the frequency change of SOAE showed that in the follicular phase SOAE migrated to a higher frequency location, and in the luteal phase SOAE migrated to lower ${ }^{[20]}$. This also highly suggests the influence of estrogen and progesterone on SOAE. Dennis ${ }^{[21]}$ compared and analyzed the differences in hearing between the sexes. The results showed that women's hearing sensitivity was higher, especially for high frequencies, the Auditory brainstem response (ABR) threshold was shorter, SOAE and Click evoked otoacoustic emissions (CEOAE) were also stronger than men. While men perform better in sound localization, binaural beat detection, and signal detection in complex masking tasks. The researchers attributed this gender difference in hearing to different hormone exposures. Our study showed that the composition 
of SSNHL typing varies among subjects in different periods. Therefore, when treating women in different periods, different treatment methods should be used to deal with the different consequences of different hormone levels. On the one hand, hormone therapy can be utilized to regulate subjects' unbalanced sex hormone levels.

On the other hand, it can also target estrogen receptor (ER) and estrogen response element (ERE) in estrogen signaling pathway to develop new treatments ${ }^{[22]}$. Studies on postmenopausal female had shown that estrogen therapy can delay their hearing loss, while progesterone had no protective effect on hearing ${ }^{[23]}$. In elderly women using hormone replacement therapy, the group using both estrogen and progesterone had worse hearing than the group using estrogen alone, and the auditory perception ability in noise environment was also lower ${ }^{[24}$, indicating that progesterone would suppress the periphery (ears) and the central (brain) auditory system, and had an antagonistic effect on estrogen ${ }^{[25]}$. Our results showed that the average hearing threshold, SSNHL severity, and typing was only significantly different between menopause and follicular phase, but not significantly different from menstrual and luteal phase. These results confirmed the protective effect of estrogen on hearing, and also suggested that there may be other complex synergies or antagonism between estrogen and progesterone, which require further research ${ }^{[26]}$.

\section{Conclusions and prospects}

This study analyzed the correlation between different physiological cycles and SSNHL in female. The results showed that the average hearing threshold of subjects in different periods was significantly different, and the average hearing threshold of subjects in follicular stage was significantly lower than that of menstrual period, luteal phase and menopause. The $\mathrm{R} \times \mathrm{C}$ table chi-square test showed that there was a correlation between subjects in different periods and the severity of SSNHL, furthur the severity of SSNHL in the follicular stage was the slightest. The analysis of SSNHL typing of subjects in different periods showed that: 1) subjects in menstrual period had more Flat descending sudden deafness and fewer High-frequency sudden deafness; 2) subjects in follicular period had more Low-frequency sudden deafness and fewer High-frequency sudden deafness; 3) subjects in luteal phase had more High-frequency sudden deafness; 4) menopause subjects had more Total sudden deafness; while, there was no significant differences in the comparison among other periods.

Our study had preliminary analyzed the incidence and severity of SSNHL in female at different periods, but there was no in-depth study on the internal mechanism and principle, nor had it completely removed the confounding factors related to mental psychology ${ }^{[27]}$. The results can explain the effect of sex hormone levels on SSNHL to a certain extent, but there were still some shortcomings remain to be improved. First, all the cases were collected from the otolaryngology electrical audiometry room. Without professional gynecologist in clinic, it was impossible to accurately determine the physiological cycle stage for each patient.All we can do was grouped the subjects by menstrual date asked by themselves. Second, because of lack of extra expense and compliance in subjects, blood samples could not be collected and accurate levels of sex hormones could not be measured. We hope to present the current preliminary results to peers in order to obtain the approval of the corresponding research structure and thus apply for funding. If we obtain financial support, we can make up for the above deficiencies, accurately assess the relationship between different physiological cycles and SSNHL, and furthur explore the correlation between sex hormone levels and SSNHL, thereby providing stronger support for the diagnosis and treatment of SSNHL.

\section{Reference}

[1] Editorial Committee of Chinese Journal of Otorhinolaryngology Head and Neck Surgery. Guidelines for Diagnosis and Treatment of Sudden Deafness (2015)[J]. Chinese Journal of Otorhinolaryngology Head and Neck Surgery, 2015, 50(6): 443-447. 
[2] Pawlak-Osinska K, Burduk P K, Kopczynski A. Episodes of repeated sudden deafness following pregnancies[J]. American Journal of Obstetrics \& Gynecology, 2009, 200(4): e7-e9.

[3] Sennaroglu G, Belgin E. Audiological findings in pregnancy[J]. Journal of Laryngology \& Otology, 2001, 115(8): 617.

[4] Yadav A, Tandon O P, Vaney N. Auditory evoked responses during different phases of menstrual cycle[J]. Indian Journal of Physiology \& Pharmacology, 2002, 46(4): 449-456.

[5] Han DY, Xiao SF. Otolaryngology Head and Neck Surgery [M]. Beijing: People's Medical Publishing House, 2016: 83-84.

[6] Teranishi M, Katayama N, Uchida Y, et al. Thirty-year trends in sudden deafness from four nationwide epidemiological surveys in Japan[J]. Acta Oto-Laryngologica, 2007, 127(12): 1259-1265.

[7] Chinese Multi-center Clinical Research Collaboration Group for Sudden Deafness. Multi-center clinical study on the classification of sudden deafness in China [J]. Chinese Journal of Otorhinolaryngology Head and Neck Surgery, 2013, 48(5): 355-361.

[8] Penido, N. O, Cruz, et al. Classification and hearing evolution of patients with sudden sensorineural hearing loss[J]. Brazilian Journal of Medical \& Biological Research, 2009, 42(8): 712-716.

[9] Torsiglieri A J, Tom L W, Keane W M, et al. Otolaryngologic manifestations of pregnancy[J]. Otolaryngology-head and neck surgery : official journal of American Academy of Otolaryngology-Head and Neck Surgery, 1990, 102(3): 293-7.

[10] Wharton J A, Church G T. Influence of menopause on the auditory brainstem response[J]. Audiology Official Organ of the International Society of Audiology, 1990, 29(4): 196-201.

[11] Yen-Pin W, Yi-Ho Y. Experience in the treatment of sudden deafness during pregnancy[J]. Acta Otolaryngol, 2006, 126(3): 271-276.

[12] Al-Mana D, Ceranic B, Djahanbakhch O, et al. Hormones and the auditory system: A review of physiology and pathophysiology[J]. Neuroscience, 2008, 153(4): 881-900.

[13] Hederstierna C, Hultcrantz M, Collins A, et al. The menopause triggers hearing decline in healthy women[J]. Hearing Research, 2010, 259(1): 31-35.

[14] Zhang C. The protective effect of estrogen on hearing organs in simulated space environment [J]. Chinese Journal of Otology, 2015, 4.

[15] Birzniece V, Bäckström T, Johansson I M, et al. Neuroactive steroid effects on cognitive functions with a focus on the serotonin and GABA systems[J]. Brain Research Reviews, 2006, 51(2): 212-239.

[16] Smith M J, Adams L F, Schmidt P J, et al. Effects of ovarian hormones on human cortical excitability[J]. Annals of Neurology, 2010, 51(5): 599-603.

[17] Wei W U, Han H L, Wang F Y, et al. Effects of estrogen on hearing organ damage caused by wind tunnel noise exposure in guinea pigs[J]. Chinese Journal of Otology, 2012.

[18] Baker M A, Weiler E M. Sex of Listener and Hormonal Correlates of Auditory Thresholds[J]. British Journal of Audiology, 1977, 11(3): 65.

[19] Frisina R D, Frisina D R. Hormone Replacement Therapy and Its Effects on Human Hearing[M]. 2016.

[20] Al-Mana D, Ceranic B, Djahanbakhch O, et al. Alteration in auditory function during the ovarian cycle[J]. Hearing Research, 2010, 268(1): 114-122.

[21] Mcfadden D. Sex differences in the auditory system[J]. Developmental Neuropsychology, 1998, 14(2-3): 261-298. 
[22] Milon B, Mitra S, Song Y, et al. The impact of biological sex on the response to noise and otoprotective therapies against acoustic injury in mice[J]. Biology of Sex Differences, 2018, 9(1): 12.

[23] Kilicdag E B, Yavuz H, Bagis T, et al. Effects of estrogen therapy on hearing in postmenopausal women[J]. American Journal of Obstetrics \& Gynecology, 2004, 190(1): 77-82.

[24] Guimaraes P, Frisina S T, Mapes F, et al. Progestin negatively affects hearing in aged women[J]. Proceedings of the National Academy of Sciences of the United States of America, 2006, 103(38): 14246.

[25] Price K, Zhu X, Guimaraes P F, et al. Hormone replacement therapy diminishes hearing in perimenopausal mice[J]. Hearing Research, 2009, 252(1): 29-36.

[26] Zhang B Y, Young Y H. Sudden Deafness during Antepartum versus Postpartum Periods[J]. ORL; journal for oto-rhino-laryngology and its related specialties, 2017, 79(5): 274.

[27] Huang HY. Analysis of psychological disorders and related factors in patients with sudden deafness [J]. Chinese Journal of Otology, 2018, 2(16).

\section{Average hearing threshold in different periods}

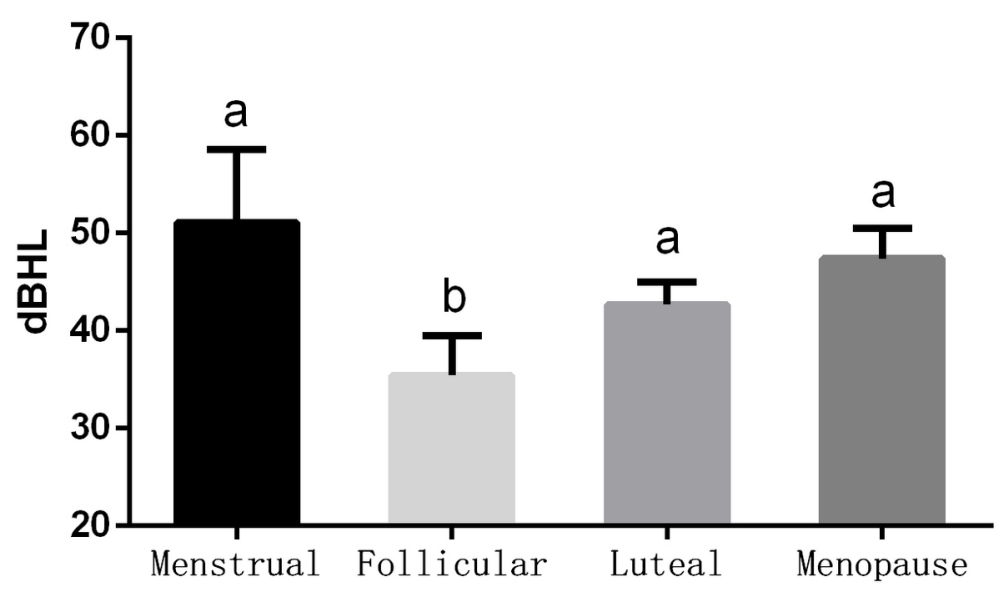

\section{Hosted file}

Table1.docx available at https://authorea.com/users/330770/articles/457577-correlation-amongdifferent-stages-of-physiological-cycles-and-sudden-sensorineural-hearing-loss-in-female

\section{Hosted file}

Table2 . docx available at https : //authorea.com/users/330770/articles/457577-correlation-amongdifferent-stages-of-physiological-cycles-and-sudden-sensorineural-hearing-loss-in-female

\section{Hosted file}

Table3. docx available at https : //authorea.com/users/330770/articles/457577-correlation-amongdifferent-stages-of-physiological-cycles-and-sudden-sensorineural-hearing-loss-in-female 